Original paper

\title{
Evaluation of mouthpiece fixation devices for head and neck radiotherapy patients fabricated in PolyJet photopolymer by a 3D printer
}

\author{
Hideki Kitamori $^{\mathrm{a}, \mathrm{c}, *}$, Iori Sumida ${ }^{\mathrm{b}, \mathrm{c}}$, Tomomi Tsujimoto ${ }^{\mathrm{c}}$, Hiroaki Shimamoto ${ }^{\mathrm{c}, \mathrm{b}}$, \\ Shumei Murakami ${ }^{\mathrm{c}, \mathrm{b}}$, Masafumi Ohki ${ }^{\mathrm{a}}$ \\ ${ }^{a}$ Department of Health Sciences, Graduate School of Medical Sciences, Kyushu University, 3-1-1 Maidashi, Higashi-ku, Fukuoka, Fukuoka 812-8582, Japan \\ ${ }^{\mathrm{b}}$ Department of Radiation Oncology, Osaka University Graduate School of Medicine, 2-2 Yamadaoka, Suita, Osaka 565-0871, Japan \\ ${ }^{\mathrm{c}}$ Department of Oral and Maxillofacial Radiology, Osaka University Graduate School of Dentistry, 1-8 Yamadaoka, Suita, Osaka 565-0871, Japan
}

\section{A R T I C L E I N F O}

\section{Keywords:}

3D printer

STL image

Mouthpiece

Head and neck radiotherapy

\begin{abstract}
A B S T R A C T
Purpose: The purpose of our study was to evaluate the usefulness of a biocompatible class VI resin PolyJet photopolymer Objet MED610 (MED610)-made mouthpiece fabricated using a 3D printer as a fixation device for head and neck radiotherapy patients.

Methods: Five mouthpieces made of GC Exafine putty type (GCEP) were fabricated from five dry skull bones. After computed tomography reconstruction of the GCEP-made mouthpiece and its surface extraction, the MED610-made mouthpieces were replicated. The sizes of the GCEP and MED610 mouthpieces were measured with a vernier caliper in width, length, and height, respectively. The volumes of these mouthpieces were measured by Archimedes' principle using pure water. For dose evaluation, the GCEP and MED610 mouthpieces were placed in the same part of a water phantom, and a 4-MV X-ray beam was located at the left maxillary gingiva, buccal mucosa, and oral floor. The dose for the planning target volume (PTV) was evaluated.

Results: The differences in the mean size and volume between the GCEP and MED610 mouthpieces were $0.03 \mathrm{~mm}$ and $0.21 \mathrm{~cm}^{3}$, respectively. Compared with the conventional GCEP mouthpiece, the dose absorption in the MED610 mouthpiece was closer to that in only water. When the mouthpiece was within the PTV margin, the minimum coverage dose at $95 \%$ of the PTV increased by $2.4 \%$ in the maxillary gingiva and by $3.6 \%$ in the buccal mucosa.

Conclusion: A 3D printer can construct a mouthpiece accurately. The MED610 mouthpiece is suitable for use in dosimetry in head and neck radiotherapy.
\end{abstract}

\section{Introduction}

Mouthpieces are widely used for occlusal fixation and tongue retraction in head and neck radiotherapy, and their usefulness has been widely reported [1-5]. Furthermore, the effectiveness of a newly developed water-equivalent mouthpiece during external beam radiotherapy for oral cancer has been described [4]. In external beam radiotherapy for cancer in the tongue, floor of the mouth, and lower gingiva, mouthpieces can reduce the radiation dose to the upper gingiva or hard palate by keeping the mouth open $[2,3]$. Mouthpieces can also immobilize the tongue, which is important when treating patients with tongue cancer using external beam radiotherapy [4,5]. Additionally, mouthpieces can reportedly reduce the radiation dose to healthy structures such as the bone tissue and the salivary glands [2-5].
Mouthpieces are also used in carbon-ion radiotherapy for head and neck tumors, and research has showed that radiation-induced mucositis can be alleviated by immobilizing the tongue with a customized mouthpiece [3].

Modeling using 3D printers has become widespread in the medical field in recent years. In the radiotherapy field, 3D printers are used for the creation of bolus [6-10], fixation devices such as shell masks $[11,12]$, and phantoms for quality assurance [13]. In studies using lowcost 3D printers, applicators for high-dose-rate brachytherapy [14], boluses [15], and radiological properties of 3D printed materials have also been reported [16]. Currently, we use mouthpieces for head and neck radiotherapy using a type of Exafine putty manufactured by the GC Co., Tokyo, Japan (GCEP) [17], which is dental impression material, for the purposes of occlusion fixation at mouth-opening position and

\footnotetext{
* Corresponding author at: Department of Oral and Maxillofacial Radiology, Osaka University Graduate School of Dentistry, 1-8 Yamadaoka, Suita, Osaka 5650871, Japan.

E-mail address: kitamori@dent.osaka-u.ac.jp (H. Kitamori).
} 


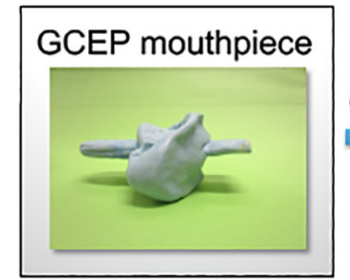

(a)

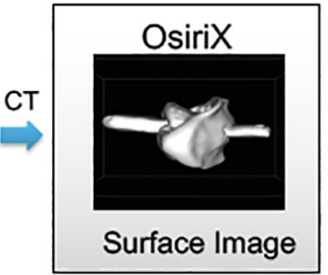

(b)

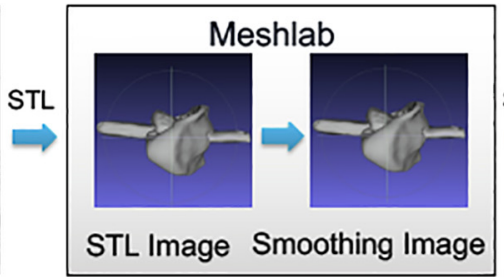

(c)

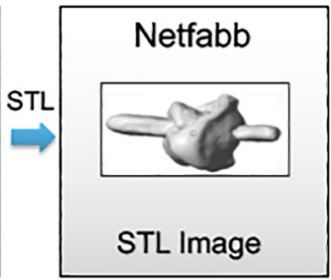

(d)

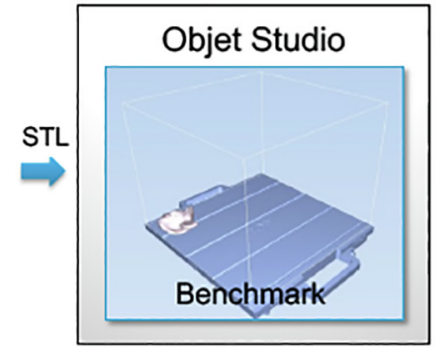

(e)

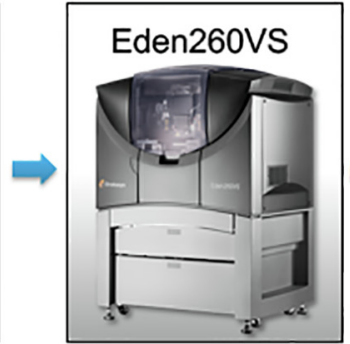

(f)

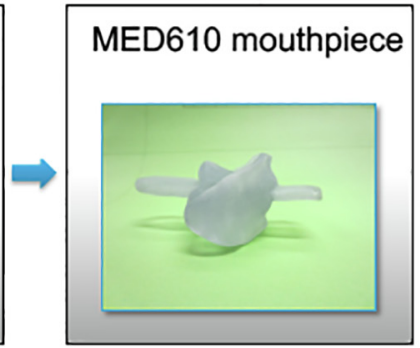

(g)

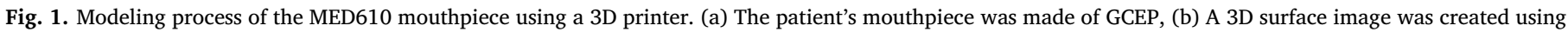

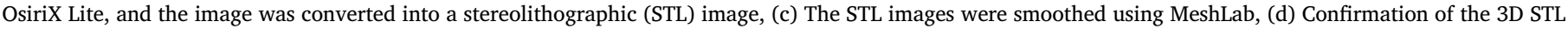

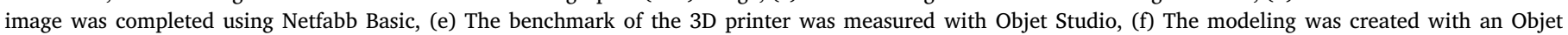
Eden260VS, (g) Completion of the MED 610 mouthpiece.
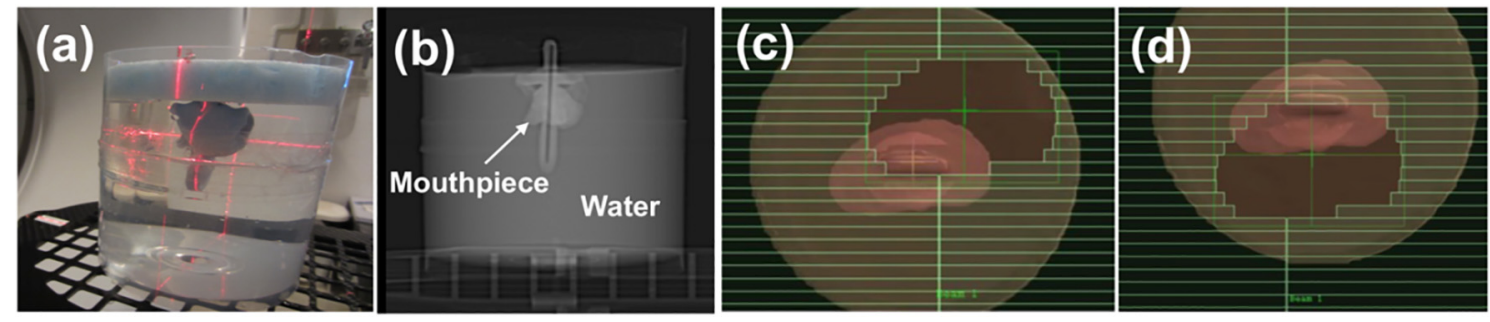

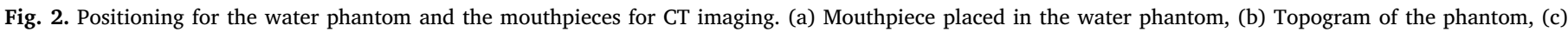
Irradiation field assuming that the beam center is at the left maxillary gingiva, (d) Irradiation field assuming that the beam center is at the oral floor.
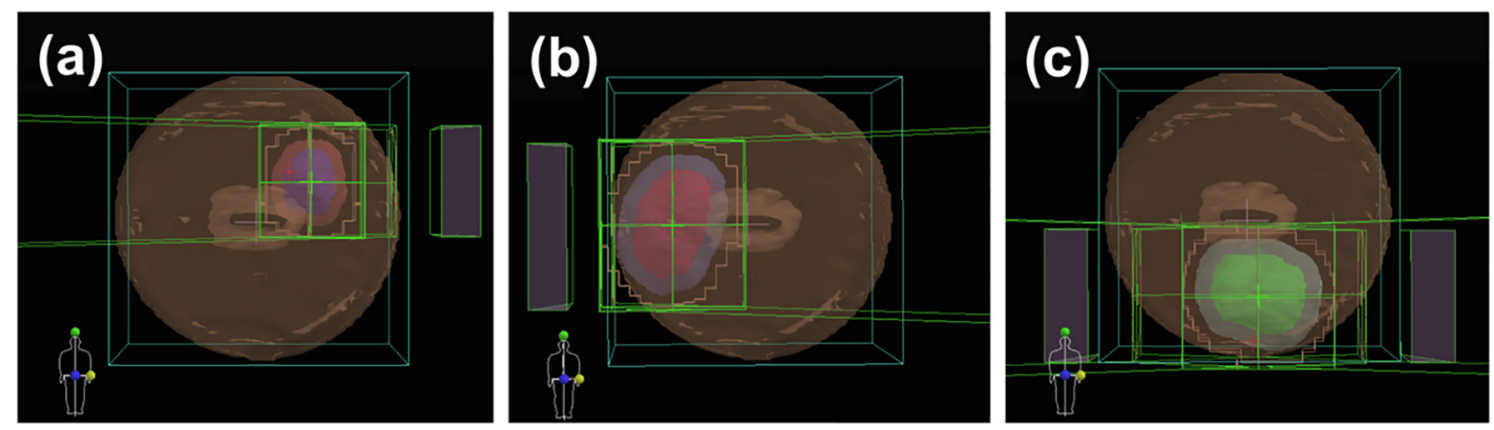

Fig. 3. Irradiation direction and number of fields to obtain the planning target volume dose of each simulated tumor margin. (a) Maxillary gingiva, (b) Buccal mucosa, (c) Oral floor.

Table 1

Measured CT value and relative electron density.

\begin{tabular}{|c|c|c|c|}
\hline \multirow[t]{2}{*}{ Material } & \multicolumn{2}{|l|}{ CT value } & \multirow{2}{*}{$\begin{array}{l}\text { Relative electron density } \\
\left(\mathrm{g} / \mathrm{cm}^{3}\right)\end{array}$} \\
\hline & Mean (HU) & $\mathrm{SD}(\mathrm{HU})$ & \\
\hline GCEP & 745.75 & 11.94 & 1.416 \\
\hline MED610 & 115.48 & 0.56 & 1.068 \\
\hline I'mRT Phantom & 2.90 & 0.14 & 0.979 \\
\hline Bolus (tissue-equivalent gel) & -4.21 & 0.20 & 0.974 \\
\hline Water & 5.92 & 0.19 & 0.981 \\
\hline
\end{tabular}

tongue immobilization. Making mouthpieces with dental material is costly in terms of the time and labor required to obtain an impression of the patient, make the gypsum model, and make the mouthpiece using resin [2-5]. GCEP mouthpieces are advantageous because they can be created directly within minutes. However, a high value of computed tomography (CT) materials such as GCEP often induces strong beam attenuation and can affect dose distribution. Therefore, we hypothesized that the biocompatible class VI resin, PolyJet photopolymer Objet MED610 (MED610), which is used as a 3D printer model resin in the oral cavity, is suitable for creating mouthpieces because of its material properties.

MED610 is a biocompatible resin that can remain in contact with 


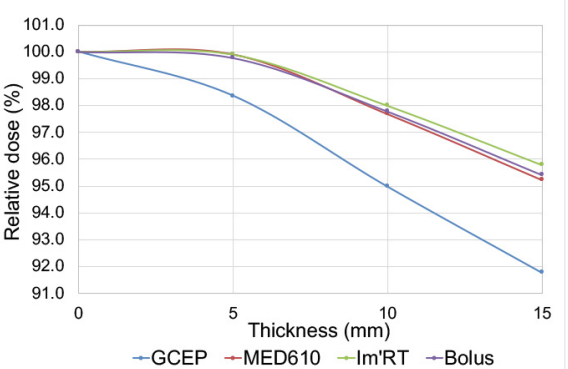

Fig. 4. Radiation dose attenuation curve of the materials tested in this experiment. GCEP: GC Exafine putty type, MED610: PolyJet photopolymer Objet MED610 modeled using a 3D printer, I'mRT: I'mRT Phantom, Bolus: Tissueequivalent gel.

the skin for more than 30 days and the mucosal membrane for up to $24 \mathrm{~h} \mathrm{[18]} \mathrm{and} \mathrm{is} \mathrm{used} \mathrm{in} \mathrm{orthopedic} \mathrm{surgery} \mathrm{[19],} \mathrm{oral} \mathrm{surgery} \mathrm{[20],} \mathrm{and}$ eye plaque brachytherapy [21]. The physical properties of MED610 are similar to those of acrylic [21].

Mouthpiece modeling using a 3D printer can be easily performed, and does not involve the need to prepare an impression using a normal dental material followed by a plaster model, and preparation with a resin.

The aim of this study was to evaluate the modeling accuracy and utility of mouthpieces modeled using MED610 with a 3D printer as fixation devices for head and neck radiotherapy patients.

\section{Methods and materials}

\subsection{Determination of the material properties}

The materials used in this experiment include GCEP and MED610, the I'mRT phantom (IBA Dosimetry GmbH, Schwarzenbruck, Germany), and tissue-equivalent gel (bolus; CIVCO, Co., Orange City, IA, USA). The CT values of the materials and the dose attenuations were measured ten times each to obtain the material properties. The CT values of the materials were measured using a LightSpeed VCT (GE Healthcare, Milwaukee, WI, USA) under the radiation therapy planning protocol with the following parameters: tube voltage, $120 \mathrm{kVp}$; tube current, $220 \mathrm{~mA}$; slice thickness, $1.25 \mathrm{~mm}$; FOV, $50 \mathrm{~cm}$. Dose attenuation of the materials were measured using a 4-MV X-ray beam from a linear accelerator (ONCOR Impression Plus, Siemens Medical Solutions,
Erlangen, Germany). The transmitted doses through the materials with different thicknesses of 5,10 , and $15 \mathrm{~mm}$ were measured using an ionchamber dosimeter (1094 type Checkmate 2; Sun Nuclear, Co., Melbourne, FL, USA). The irradiation field was $2 \mathrm{~cm} \times 2 \mathrm{~cm}$, and a dose of 200 monitor units (MU) was irradiated at a dose rate of $200 \mathrm{MU} / \mathrm{min}$ using a 4-MV X-ray.

\subsection{Modeling the MED610 mouthpiece using a 3D printer}

Fig. 1 shows the process for modeling the MED610 mouthpiece using a 3D printer. First, the patient's mouthpiece was made using GCEP, and then CT imaging of the mouthpiece was performed under the following conditions: FOV, $9.6 \mathrm{~cm}$; slice thickness, $0.625 \mathrm{~mm}$. A 3D surface image was created using OsiriX Lite (v.8.0.1; Pixmeo Co., Geneva, Switzerland) software [22], and the image was converted into a stereolithographic (STL) image. Next, the STL images were smoothed using MeshLab 64-bit (v.1.3.3; Visual Computing Lab - ISTI - CNR) software [23], and we confirmed the completed 3D STL images using Netfabb Basic 5.2 (C) Autodesk, Inc., San Rafael, CA, USA) software [24]. The benchmark of the 3D printer was measured with Objet Studio (Stratasys Ltd., NY, USA) software [25,26], and modeling was performed with an Objet Eden260VS (Stratasys Ltd. NY, USA) 3D printer [26] at $30-\mu \mathrm{m}$ resolution.

Concavo-convex parts are formed where the mouthpiece made with GCEP meshes over the teeth. Although GCEP is elastic, MED610 is not. Thus, we have considered the Laplacian smoothing on STL 3D images to improve tooth engagement.

We evaluated whether the FOV of the CT images influenced the resolution of the 3D STL image of the mouthpieces by image observation prepared using three FOVs: $25 \mathrm{~cm}, 18 \mathrm{~cm}$, and $9.6 \mathrm{~cm}$.

\subsection{Correlation between the change in size and volume and the image- processing coefficients}

To investigate the change in the 3D surface, the threshold value, the thinning factor, the flattening coefficient, and the resolution coefficient were modified using OsiriX, and the STL data were imported into MeshLab. Subsequently, a 3D surface STL image was created by changing the Laplacian smoothing coefficient. We acquired the size and volume on the 3D surface STL image using Netfabb Basic 5.2 (@) Autodesk, Inc.) software. The results for five GCEP mouthpieces were obtained using relative values to the maximum size and the volume of the STL images.
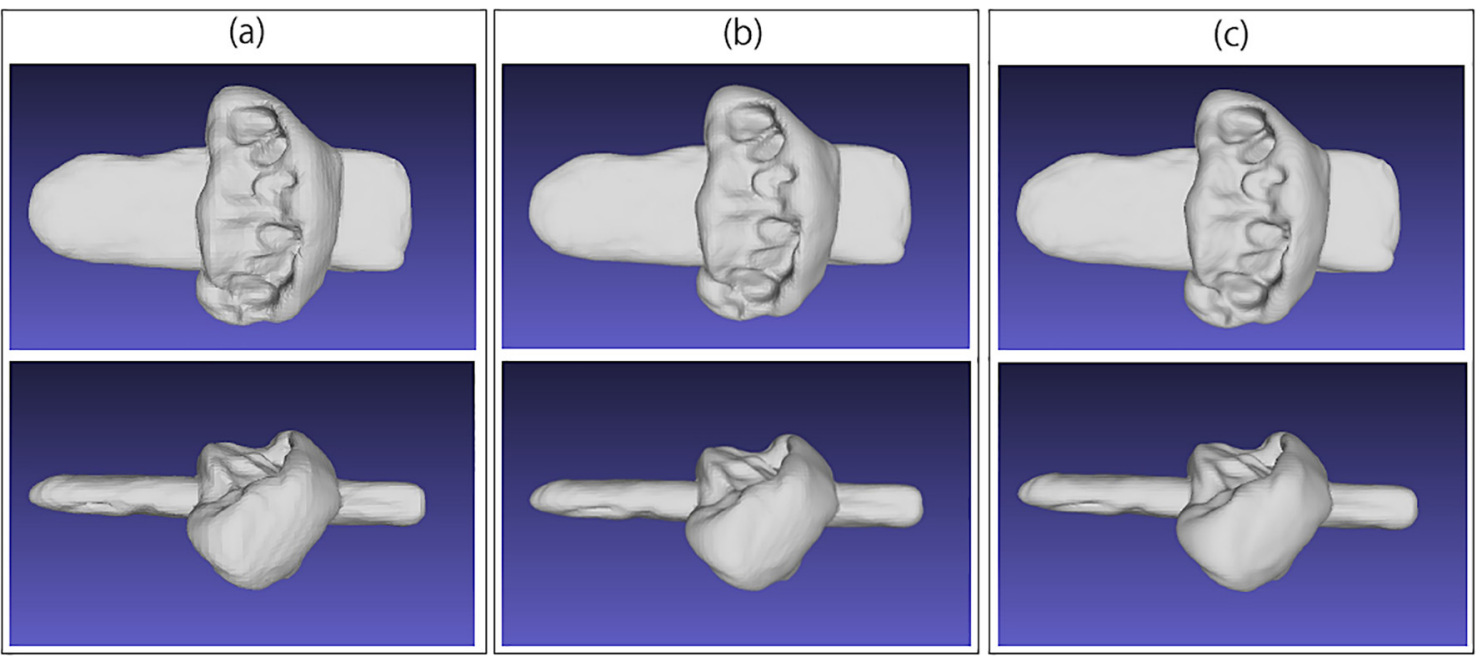

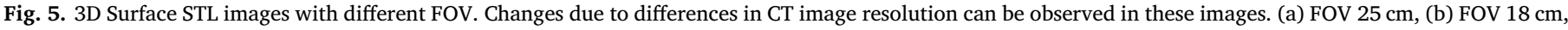
(c) FOV $9.6 \mathrm{~cm}$ 
(a)
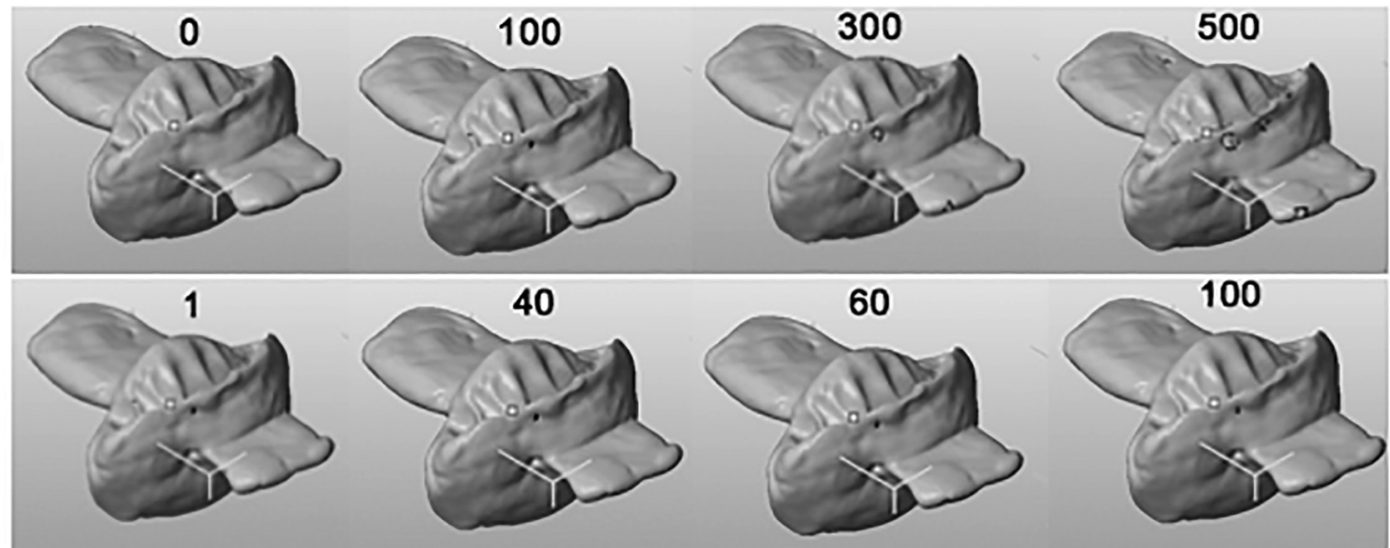

(c)
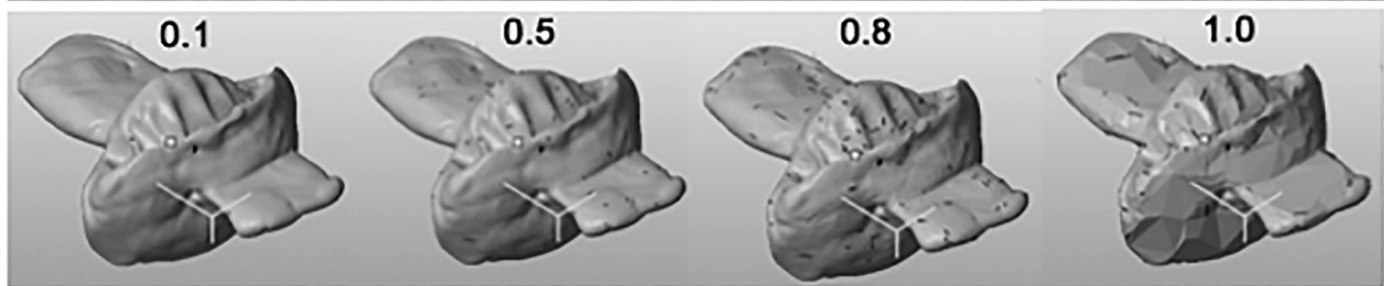

(d)
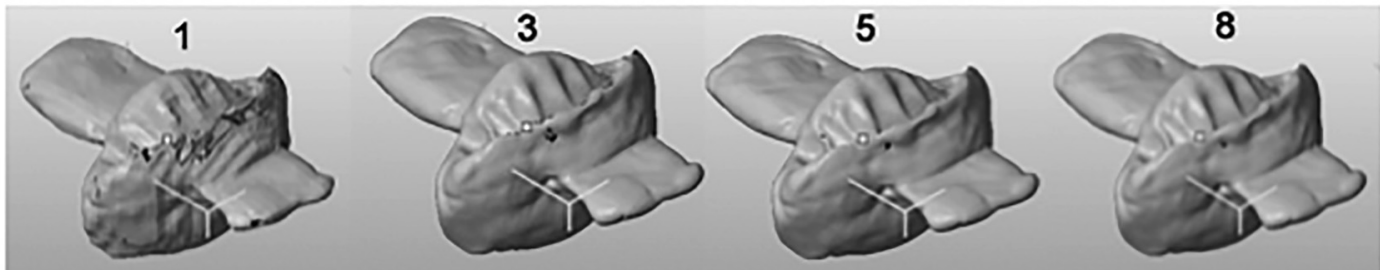

(e)
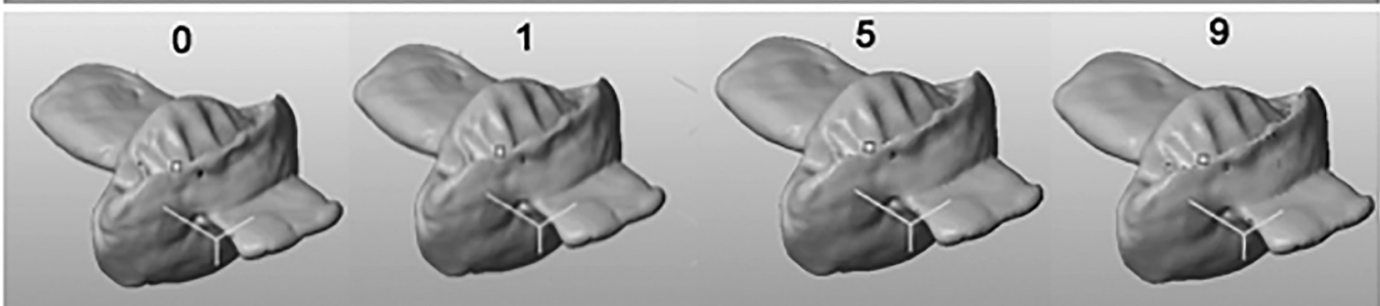

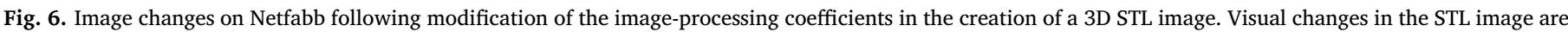

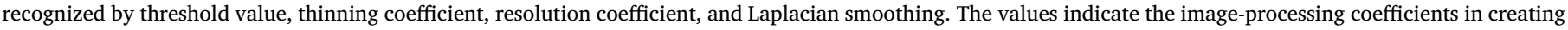

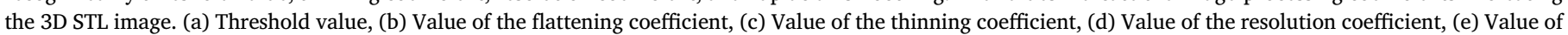
the Laplacian smoothing coefficient.

\subsection{The size and volume differences between the GCEP and MED610 mouthpieces}

The sizes of the GCEP and MED610 mouthpieces were measured using a digital vernier caliper in width, length, and height, respectively. The actual volumes for the two materials were measured by Archimedes' principle using pure water. Each measurement was performed ten times. The mean size and volume of each of the GCEP and MED610 mouthpieces was determined, and the mean values were statistically analyzed using a paired $t$-test to test for significant differences.

\subsection{Dose distribution with a single-beam}

For dose evaluation, each GCEP and MED610 mouthpiece was placed at the same position in a water phantom, and CT imaging was taken at the following acquisition settings (Fig. 2). The phantom was scanned using a LightSpeed VCT (GE Healthcare) under the standard radiation therapy planning protocol: tube voltage, $120 \mathrm{kVp}$; tube current, $220 \mathrm{~mA}$; slice thickness, $1.25 \mathrm{~mm}$; FOV, $50 \mathrm{~cm}$. All CT data were imported into a XiO (v.4.50; Elekta, Stockholm, Sweden) treatment planning system, and a single anterior-posterior beam plan for a 4-MV $\mathrm{X}$-ray beam was set in the system, assuming that the beam center was at the maxillary gingiva or oral floor. The dose distribution in each case was evaluated in the axial and sagittal planes.

\subsection{Dose-volume histogram for the planning target volume}

A simulated tumor margin was set up, and a clinical beam setting was performed with the XiO treatment planning system. The simulated tumor margins were assigned at the maxillary gingiva, buccal mucosa, and oral cavity. The dose-volume histogram was used to evaluate the minimum coverage dose of $95 \%$ of the planning target volume (PTV) of each simulated tumor margin. The prescribed dose was $200 \mathrm{cGy}$. Similar to clinical treatment regimens, the irradiation fields of two wedge pairs were planned for the maxillary gingiva and buccal mucosa, and three fields were planned for the oral floor (Fig. 3). 
(a)

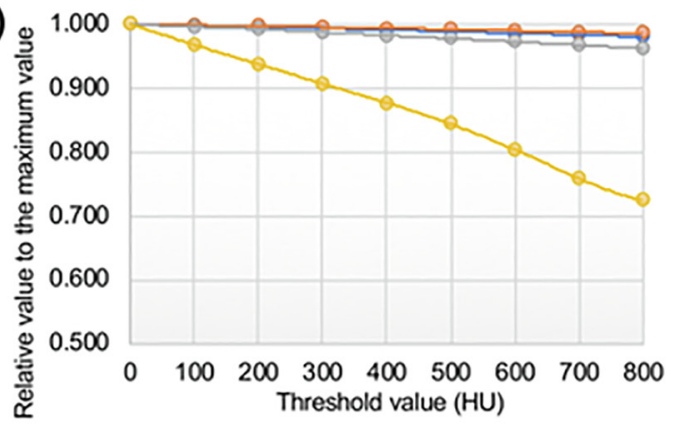

(c)

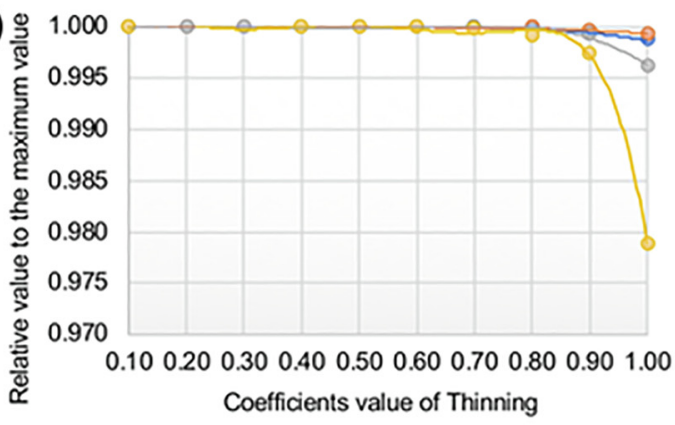

(e)

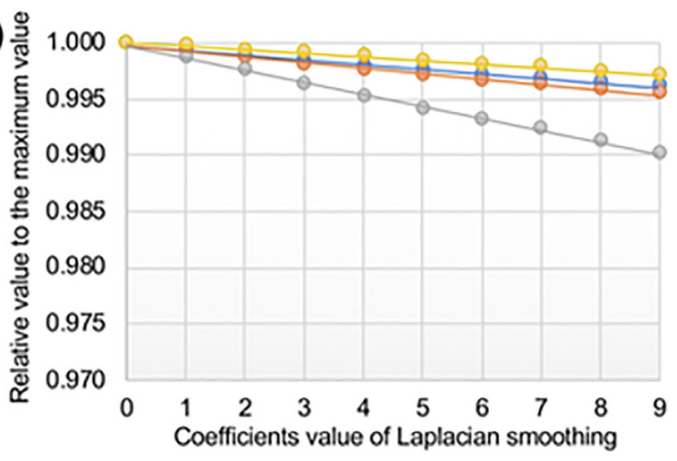

(b)

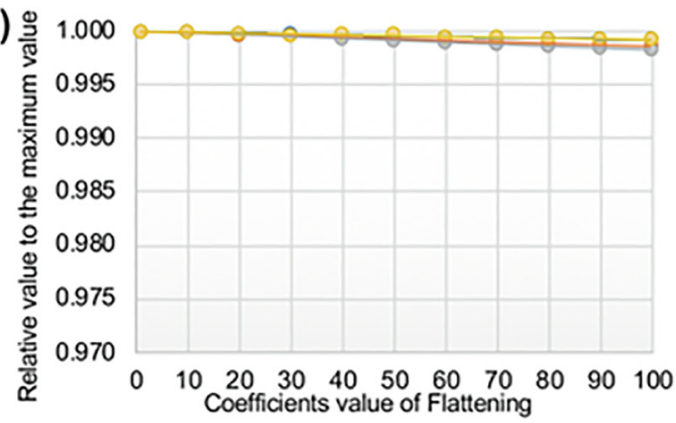

(d)

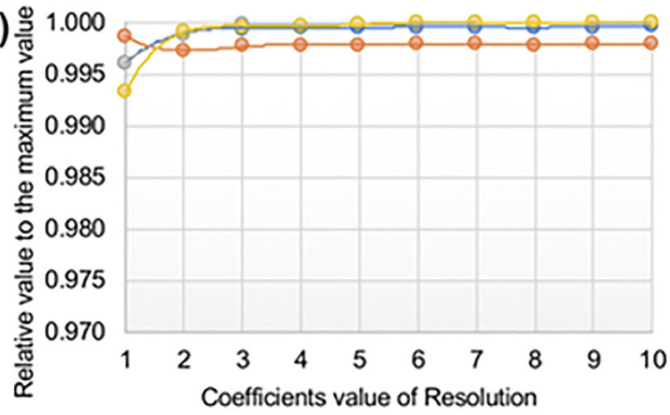

- Width (mm)

- Length (mm)

- Height $(\mathrm{mm})$

- Volume $\left(\mathrm{cm}^{3}\right)$

Fig. 7. Change in size and volume because of changes in the image-processing coefficients in creating the creation of a 3D image of a surface. The vertical axis shows the mean value of the relative-maximum value of the five mouthpieces. The horizontal axis shows the coefficient of each processing condition. (a) Threshold value, (b) Value of the flattening coefficient, (c) Value of the thinning coefficient, (d) Value of the resolution coefficient, (e) Value of the Laplacian smoothing coefficient.

\begin{tabular}{|c|c|c|c|c|c|c|}
\hline \multirow{2}{*}{ Irradiation site } & \multicolumn{3}{|c|}{ Axial plane } & \multicolumn{3}{c|}{ Sagittal plane } \\
\cline { 2 - 7 } & GCEP & MED610 & Water & GCEP & MED610 & Water \\
\hline $\begin{array}{c}\text { Mangillary } \\
\text { gingiva }\end{array}$ & & & & \\
\hline
\end{tabular}

Dose values(\%) 10710510095908580706050

Fig. 8. Single-beam dose distribution. The dose distribution for the MED610 mouthpiece was close to that in water. GCEP: GC Exafine putty type, MED610: PolyJet photopolymer Objet MED610 modeled using a 3D printer. 
Table 2

Mean size and volume of the GCEP and MED610 mouthpieces.

\begin{tabular}{|c|c|c|c|c|}
\hline \multicolumn{2}{|c|}{ Mouthpiece No. } & \multicolumn{2}{|l|}{ Material } & \multirow[t]{2}{*}{ P-value } \\
\hline & & GCEP & MED610 & \\
\hline \multirow[t]{4}{*}{1} & Width (mm) & $47.39 \pm 0.02$ & $47.37 \pm 0.03$ & 0.1198 \\
\hline & Length (mm) & $72.88 \pm 0.02$ & $72.87 \pm 0.02$ & 0.1039 \\
\hline & Height (mm) & $29.10 \pm 0.02$ & $29.08 \pm 0.02$ & 0.1003 \\
\hline & Volume $\left(\mathrm{cm}^{3}\right)$ & $20.07 \pm 0.20$ & $19.94 \pm 0.24$ & 0.1075 \\
\hline \multirow[t]{4}{*}{2} & Width (mm) & $50.73 \pm 0.02$ & $50.71 \pm 0.02$ & 0.1206 \\
\hline & Length (mm) & $73.82 \pm 0.02$ & $73.80 \pm 0.02$ & 0.0786 \\
\hline & Height (mm) & $28.52 \pm 0.02$ & $28.50 \pm 0.02$ & 0.0744 \\
\hline & Volume $\left(\mathrm{cm}^{3}\right)$ & $21.41 \pm 0.22$ & $21.28 \pm 0.19$ & 0.1552 \\
\hline \multirow[t]{4}{*}{3} & Width (mm) & $50.80 \pm 0.02$ & $50.79 \pm 0.02$ & 0.0740 \\
\hline & Length (mm) & $78.26 \pm 0.02$ & $78.24 \pm 0.02$ & 0.0902 \\
\hline & Height (mm) & $31.53 \pm 0.02$ & $31.51 \pm 0.02$ & 0.1341 \\
\hline & Volume $\left(\mathrm{cm}^{3}\right)$ & $21.61 \pm 0.19$ & $21.47 \pm 0.21$ & 0.0776 \\
\hline \multirow[t]{4}{*}{4} & Width (mm) & $52.22 \pm 0.03$ & $52.20 \pm 0.02$ & 0.0751 \\
\hline & Length (mm) & $74.24 \pm 0.02$ & $74.23 \pm 0.02$ & 0.0997 \\
\hline & Height (mm) & $29.72 \pm 0.02$ & $29.70 \pm 0.02$ & 0.0943 \\
\hline & Volume $\left(\mathrm{cm}^{3}\right)$ & $23.22 \pm 0.23$ & $23.07 \pm 0.21$ & 0.0701 \\
\hline \multirow[t]{4}{*}{5} & Width (mm) & $52.04 \pm 0.03$ & $52.02 \pm 0.02$ & 0.1059 \\
\hline & Length (mm) & $74.94 \pm 0.02$ & $74.93 \pm 0.02$ & 0.1031 \\
\hline & Height (mm) & $26.60 \pm 0.03$ & $26.58 \pm 0.02$ & 0.0737 \\
\hline & Volume $\left(\mathrm{cm}^{3}\right)$ & $20.85 \pm 0.23$ & $20.70 \pm 0.22$ & 0.0920 \\
\hline
\end{tabular}

Table 3

Differences in the size and volume between the GCEP and MED610 mouthpieces.

\begin{tabular}{lcccc}
\hline Mouthpiece No. & Width $(\mathrm{mm})$ & Length $(\mathrm{mm})$ & Height $(\mathrm{mm})$ & Volume $\left(\mathrm{cm}^{3}\right)$ \\
\hline 1 & $0.03 \pm 0.02$ & $0.02 \pm 0.01$ & $0.03 \pm 0.02$ & $0.23 \pm 0.11$ \\
2 & $0.03 \pm 0.02$ & $0.02 \pm 0.02$ & $0.02 \pm 0.02$ & $0.21 \pm 0.19$ \\
3 & $0.02 \pm 0.02$ & $0.03 \pm 0.02$ & $0.02 \pm 0.02$ & $0.21 \pm 0.17$ \\
4 & $0.03 \pm 0.03$ & $0.02 \pm 0.01$ & $0.03 \pm 0.03$ & $0.21 \pm 0.19$ \\
5 & $0.03 \pm 0.03$ & $0.03 \pm 0.02$ & $0.03 \pm 0.03$ & $0.22 \pm 0.19$ \\
Mean & $0.03 \pm 0.02$ & $0.03 \pm 0.02$ & $0.03 \pm 0.02$ & $0.21 \pm 0.17$ \\
\hline
\end{tabular}

\section{Results}

\subsection{Determination of the material properties}

The CT values of GCEP and MED610 were $745.75 \pm 11.94 \mathrm{HU}$ and $115.48 \pm 0.56 \mathrm{HU}$, respectively. The electron density as assessed using the CT values electron density conversion table was $1.416 \mathrm{~g} / \mathrm{cm}^{3}$ for GCEP and $1.068 \mathrm{~g} / \mathrm{cm}^{3}$ for MED610 (Table 1). As an effect of internal uniformity, the GCEP's Standard Deviation (SD) was considered to be larger compared to other materials.

The radiation dose attenuation of MED610 was close to that of the bolus (tissue-equivalent gel) and the I'mRT phantom. GCEP showed the largest dose attenuation among the materials tested in this experiment (Fig. 4).

\subsection{Modeling of the MED610 mouthpiece using a 3D printer}

Using our modeling method, it was possible to create 3D surface images with free software, OsiriX. Once suitable image-processing conditions were established, an appropriate model could be created. It was necessary to set the FOV as small as possible because the resolution of the 3D STL image was affected by the voxel size of the CT image (Fig. 5). The phenomenon of this image was similar in each of the five mouthpieces.
3.3. Correlation between the change in size and volume of the STL image and the change in the image-processing coefficients

First, we visually observed the 3D STL image on Netfabb (Fig. 6). As the threshold value increased, a part of the mouthpiece image disappeared (Fig. 6a). Changes in flattening coefficient did not produce much of an effect (Fig. 6b). When the thinning value increased, the prototype of the mouthpiece became rough and distorted (Fig. 6c). When the resolution value was low, the prototype of the mouthpiece was found lacking and distorted. However, there was not much change when the resolution value was 5 or higher (Fig. 6d). As the value of the Laplacian smoothing value increased, the surface became smoother (Fig. 6e).

Next, we examined changes in the size and volume on the 3D STL image when the image-processing conditions were changed (Fig. 7).

The vertical axis in Fig. 7 shows the mean value of the relative value to the maximum value of the five mouthpieces. The horizontal axis shows the coefficient of each processing condition. Changing the threshold value affected the size and volume (Fig. 7a); however, the flattening (Fig. 7b) and thinning coefficients (Fig. 7c) did not affect the size and volume when they were small. The resolution was constant when the coefficient was $\geq 5$ (Fig. $7 \mathrm{~d}$ ). The Laplacian smoothing value affected the size and volume (Fig. 7e).

Based on image confirmation of the 3D STL image by Netfabb as shown in Fig. 7 and the changes in size and volume on the 3D STL image as shown in Fig. 8, we determined the optimal image-processing conditions to be as follows: threshold value, 100; flattening coefficient, 1.0 ; thinning coefficient, 0.1 ; resolution coefficient, $\geq 5$; Laplacian smoothing coefficient, 1.0.

We generated 3D STL images using this image-processing condition for all five mouthpieces and created MED610 mouthpieces using the Objet Eden260VS 3D printer.

\subsection{Difference in size and volume of the GCEP and MED610 mouthpieces}

The size and volume of the 3D STL images produced using the optimal image-processing conditions were determined by Netfabb. The differences in size and volume between the GCEP and MED610 mouthpieces were small and not significant in the five mouthpieces made of each of the two materials (Table 2).

The average of the differences in size and volume of the GCEP and MED610 mouthpieces was $30 \mu \mathrm{m}$ and $0.21 \mathrm{~cm}^{3}$, respectively (Table 3).

\subsection{Single-beam dose distribution}

Fig. 8 shows 2D dose distributions at the maxillary gingiva and oral floor. The dose distribution for the MED610 mouthpiece was close to that in water and better than that for the GCEP mouthpiece (Fig. 8).

\subsection{Dose-volume histogram for the planning target volume}

When the mouthpiece was included near the simulated tumor margin, the dose-volume histogram of the MED610 mouthpiece was better than that of the GCEP mouthpiece. Differences were found in the maxillary gingiva and buccal mucosa; the least difference was found in the oral floor (Fig. 9). In evaluating the minimum coverage dose of $95 \%$ of the PTV using the MED610 and GCEP mouthpieces, the maxillary gingiva was $188.9 \mathrm{cGy}$ and $184.5 \mathrm{cGy}$, respectively, whereas the oral mucosa was $191.1 \mathrm{cGy}$ and $184.4 \mathrm{cGy}$, respectively. No difference was seen between the mouthpieces at the oral floor at a dose of $193.3 \mathrm{cGy}$.

\section{Discussion}

In this study, we evaluated the usefulness of a MED610 mouthpiece modeled using a 3D printer as a fixation device for head and neck radiotherapy patients. The image-processing conditions were evaluated 

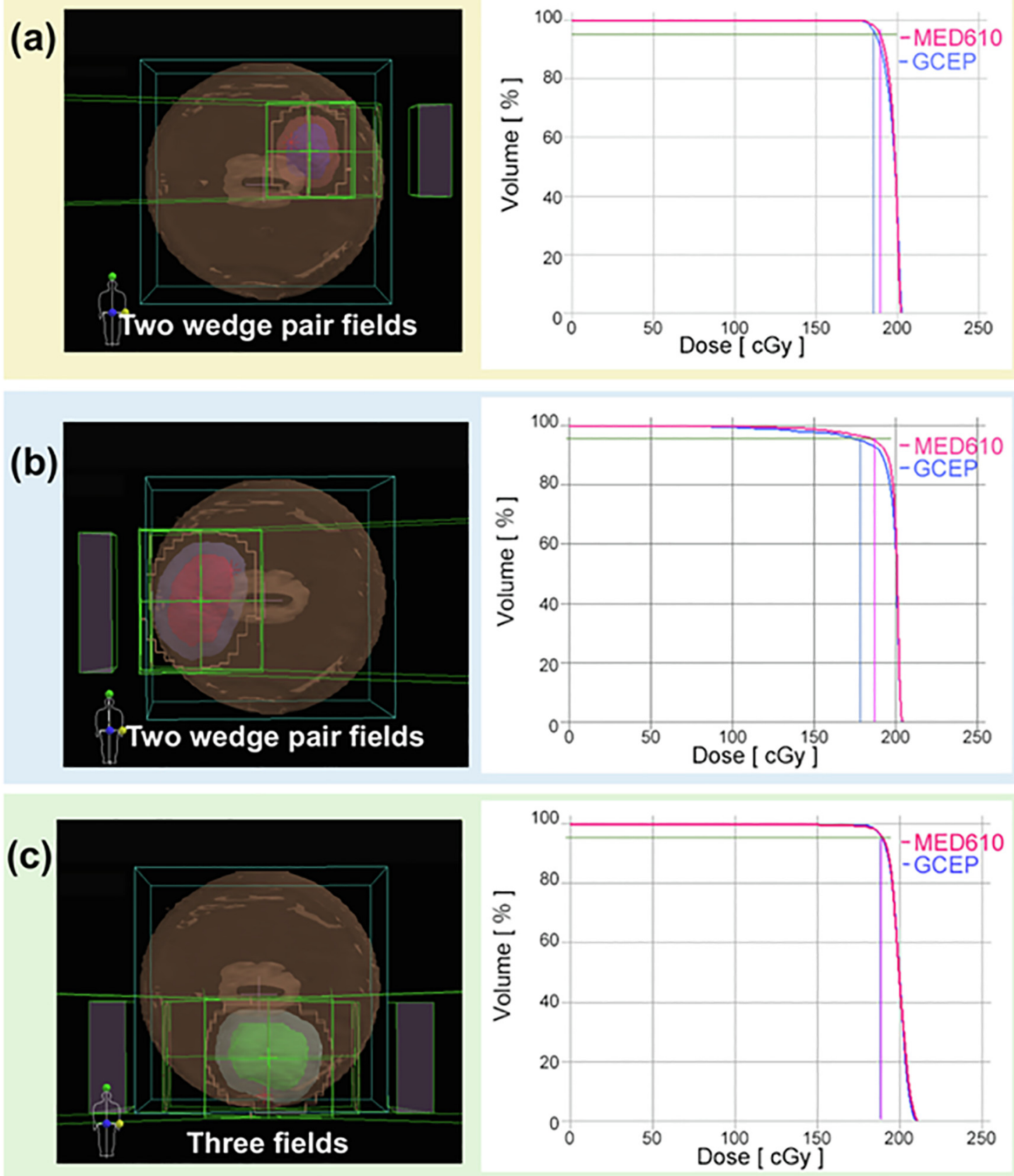

Fig. 9. Dose-volume histogram for the planning target volume. (a) Maxillary gingiva, (b) Buccal mucosa, (c) Oral floor GCEP: GC Exafine putty type, MED610: PolyJet photopolymer Objet MED610 modeled using a 3D printer.

with OsiriX, which showed that the differences in the size and volume of the GCEP and MED610 mouthpieces were $30 \mu \mathrm{m}$ and $0.21 \mathrm{~cm}^{3}$, respectively (Table 3$)$.

The Eden260VS 3D printer has an uncertainty [25] of $20-85 \mu \mathrm{m}$ in size $50 \mathrm{~mm}$ and the uncertainty measured in the present study falls within this range.

Ricotti $\mathrm{R}$ et al. evaluated an applicator printed in 3D with acrylonitrile butadiene styrene at various packing rates for high-dose-rate proximity irradiation. They reported that printing accuracy using a lowcost 3D printer was within $0.5 \mathrm{~mm}$ [14]. For application to bolus formation, we studied an applicator printed in 3D with acrylonitrile butadiene styrene and polylactide at various filling rates, and the structure of Borus is depicted using iPlan TPS, guaranteeing a perfect fit to the body surface. Reports have shown that the density (HU value) of the defined bolus can be printed using superficial coverage and bolus at a filling rate according to the calibration curve. The time required for bolus printing increases with the increasing packing rate, which takes approximately $150 \mathrm{~min}$ at a $10 \%$ fill rate and approximately $330 \mathrm{~min}$ at a $60 \%$ rate [15]. Dancewicz at al. reports that it is possible to construct useful phantoms by utilizing 3D printers with different filling rates to model various body tissues and for use in both imaging and dosimetry [16]. We used a PolyJet type printer for our work, so we could not change the resin filling rate. With reference to the CT value - electron density conversion table [27], the electron density of MED610 may be considered similar to the liver, and so we obtain more precise results.

Because the resolution of the 3D STL image is influenced by the voxel size, it was necessary to set the FOV as small as possible (Fig. 5). The difference in the 3D STL images by FOV is $0.488 \times 0.488 \times 0.625 \mathrm{~mm}^{3}$ for a voxel size of FOV $25 \mathrm{~cm}$, $0.351 \times 0.351 \times 0.625 \mathrm{~mm}^{3}$ for a voxel size of $18 \mathrm{~cm} \mathrm{FOV}$, and 

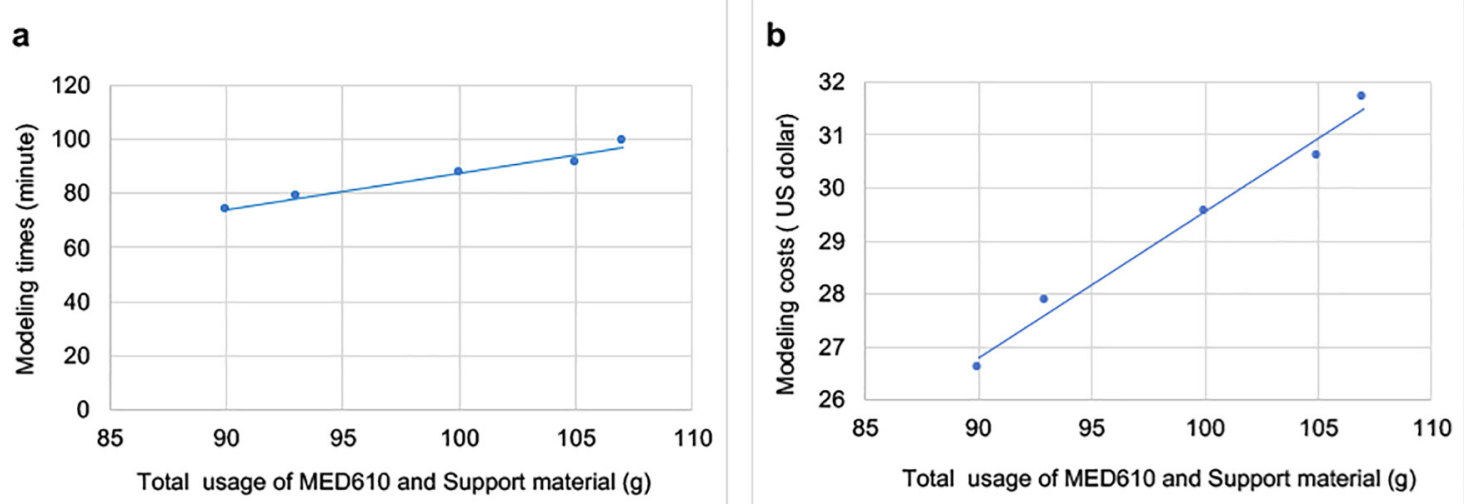

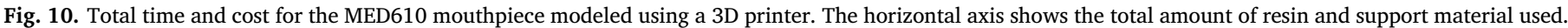
(a) Modeling time with 3D printer, (b) Modeling cost.

$0.188 \times 0.188 \times 0.625 \mathrm{~mm}^{3}$ for a voxel size of $9.6 \mathrm{~cm}$ FOV. This spatial resolution affects 3D STL images. Since 3D printers faithfully reproduce 3D STL, it is necessary to obtain original, high-resolution CT images.

We created a 3D STL image using the CT image of the mouthpiece so that it can be displayed on CT imaging even where the depressed concave portion of the concavo-convex portion of the teeth of the GCEP mouthpiece is not visible from the surface.

Regarding the use of a mouthpiece for head and neck radiotherapy, Wagner et al. reported dose uncertainty caused by the daily change of the step bite block position during dynamic types of treatment such as intensity-modulated radiation therapy (IMRT) [1]. However, they reported that step bite blocks should not be used because it is difficult to accurately position them the same way with every use. Verrone et al. recommended the use of an intraoral stent device to reduce the radiation dose to adjacent structures such as the bone tissue and salivary glands during radiotherapy [2]. Musha et al. reported decreased radiation-induced mucositis with the use of a mouth-opening device for carbon-ion radiotherapy that was similar to the device reported by Verrone et al [3]. Furthermore, Kudoh et al. reported the usefulness of mouthpieces in external beam radiation therapy for oral cancer [4]; their method is considered to be effective because it improves the nonuniformity correction by using a water-equivalent material. However, because there was no protrusion at the tip of their mouthpiece, facial movement was possible within the shell. We concluded that mouthpieces designed to be fixed by dental arches can promote superior dose distribution as well as achieve tongue immobilization in oral cancer patients. The entire process of our modeling method takes about $1 \mathrm{~h}$. The mouthpieces made in this study were fixed by the upper and lower dental arches, with an open-mouth position, and the tongue can be fixed and the mouthpiece set at the same position each day. Therefore, the tongue and jaw position can be kept constant in 3D conformal radiotherapy (3D-CRT) and IMRT.

Although the GCEP mouthpiece is easy to fabricate, it introduces a dose attenuation caused by the high CT value of the material as reflected in the results of the single-beam dose distribution and dose-volume histogram for the PTV. The MED610 mouthpiece resulted in uniform dose distribution and showed a favorable dose-volume histogram for the PTV.

From the evaluation of the minimum coverage dose of $95 \%$ of the PTV at the three sites, when the mouthpiece was within the PTV margin, the minimum coverage dose was able to obtain a dose increase of $2.4 \%$ in the maxillary gingiva and $3.6 \%$ in the buccal mucosa. The overlap between the PTV and the mouthpiece in the oral floor was small, and the influence of the mouthpiece material on the dose was reduced by multi-directional irradiation (Fig. 9).

Shimamoto et al. evaluated the scatter doses in the direction of the buccal mucosa from dental metals. The scatter dose from the dental metals was 1.4-6.9\% for 3D-CRT and 1.4-4.3\% for IMRT, and both fall within the measurement uncertainty [28]. They reported that the distance of influence of the scatter dose from dental gold was theoretically less than $8 \mathrm{~mm}$. Therefore, the depth of $22 \mathrm{~mm}$, i.e., at a distance of $8 \mathrm{~mm}$ from the dental metal, is regarded as an appropriate normalized position, and if this distance is used, the influence of the scattered ray can be prevented. We believe that scattered radiation can be absorbed by the thickness of MED610 instead of distance.

The thickness of the buccal side of the GCEP mouthpiece, which was used for an actual patient, was about $8 \mathrm{~mm}$ at the maxillary molar region and about $10 \mathrm{~mm}$ at the mandibular molar region. It is possible to reduce the influence of scattered radiation from the dental metal in the MED610 mouthpiece to the buccal mucosa. From the radiation dose attenuation curve for MED610 in Fig. 4, the dose was estimated to decrease by $1.5 \%$ in the direction of the buccal side in the maxilla and by $2.5 \%$ in the mandible, respectively.

Expert skill is required when creating impressions using normal dental materials. Creating mouthpieces using a 3D printer requires time and labor; however, when the 3D STL image of the mouthpiece is completed, the 3D printer can create the shape automatically. Therefore, 3D-printed mouthpieces can be created even by those who are not a dentist or dental technician.

The total time and cost for in-hospital modeling of the MED610 mouthpiece on the 3D printer are shown for reference in Fig. 10. In Japan, since 2017, 10,000 yen can be claimed from insurance for mouthpieces used for prognosing mucositis. However, the burden of out of pocket cost to the patient is only $10 \%-30 \%$ of that amount. The involvement of a professional contractor for the modeling will cost approximately 4 times as much as the creation fee and will take approximately one week to deliver. Model printing in the hospital can be successfully accomplished using the time and expense outlined in Fig. 10.

We used the EDEN 260 VS 3D printer for modeling, but MED610 can be used with other PolyJet type 3D printers.

\section{Conclusions}

Computer modelling and fabrication of mouthpieces using a 3D printer using MED610 polymer resin resulted in high accuracy in terms of size and volume under the optimal image-processing conditions that we established. Dose absorption by the MED610 mouthpiece was also lower than with the conventional GCEP mouthpiece, and the dose distribution and dose-volume histogram were also better in the former. Therefore, the MED610 mouthpiece is suitable for use as a fixation device in head and neck radiotherapy. 


\section{Acknowledgements}

We appreciate Mr. Hideki Goto of Japan Cannon Lifecare Solutions Co., Ltd who advised in this research.

\section{Conflicts of interest}

The authors have no relevant conflicts of interest to disclose.

\section{References}

[1] Wagner D, Anton M, Vorwerk H. Dose uncertainty in radiotherapy of patients with head and neck cancer measured by in vivo ESR/alanine dosimetry using a mouthpiece. Phys Med Biol 2011;56(5):1373-83. https://doi.org/10.1088/0031-9155/ 56/5/010.

[2] Verrone JR, Alves Fde A, Prado JD, Boccaletti KW, Sereno MP, Silva ML, et al. Impact of intraoral stent on the side effects of radiotherapy for oral cancer. Head Neck 2013;35(7):E213-7. https://doi.org/10.1002/hed.23028.

[3] Musha A, Saitoh J, Shirai K, Kubota Y, Shimada H, Abe T, et al. Customized mouthpieces designed to reduce tongue mucositis in carbon-ion radiotherapy for tumors of the nasal and paranasal sinuses. Phys Imaging Radiat Oncol 2017;3:1-4. https://doi.org/10.1016/j.phro.2017.07.003.

[4] Kudoh T, Ikushima H, Kudoh K, Furutani S, Kawanaka T, Kubo A, et al. Effectiveness of newly developed water-equivalent mouthpiece during external beam radiotherapy for oral cancer. Ann Carcinog 2017;2(1):1007.

[5] Doi H, Tanooka M, Ishida T, Moridera K, Ichimiya K, Tarutani K, et al. Utility of intraoral stents in external beam radiotherapy for head and neck cancer. Rep Pract Oncol Radiother 2017;22(4):310-8. https://doi.org/10.1016/j.rpor.2017.03.002.

[6] Kim SW, Shin HJ, Kay CS, Son SH. A customized bolus produced using a 3-dimensional printer for radiotherapy. PLoS ONE 2014;9(10):e110746https://doi.org/ 10.1371/journal.pone.0110746.

[7] Lukowiak M, Boehlke M, Matias D, Jezierska K, Piatek-Hnat M, Lewocki M, et al. Use of a 3D printer to create a bolus for patients undergoing tele-radiotherapy. Int $J$ Radiat Res 2017;14(4):287-95https://doi.org/10.18869/acadpub.ijrr.14.4.287.

[8] Su S, Moran K, Robar JL. Design and production of 3D printed bolus for electron radiation therapy. J Appl Clin Med Phys 2014;15(4):4831. https://doi.org/10. 1120/jacmp.v15i4.4831.

[9] Zou W, Fisher T, Kim L, Kim L, Chen T, Narra V, et al. Potential of 3D printing technologies for fabrication of electron bolus and proton compensators. J Appl Clin Med Phys 2015;16(3):4959. https://doi.org/10.1120/jacmp.v16i3.4959.

[10] Lukowiak M, Jezierska K, Boehlke M, Więcko M, Łukowiak A, Podraza W, et al. Utilization of a 3D printer to fabricate boluses used for electron therapy of skin lesions of the eye canthi. J Appl Clin Med Phys 2017;18(1):76-81. https://doi.org/ $10.1002 / \mathrm{acm} 2.12013$.

[11] Laycock SD, Hulse H, Scrase CD, Tam MD, Isherwood S, Mortimore DB, et al. Towards the production of radiotherapy treatment shells on 3D printers using data derived from DICOM CT and MRI: preclinical feasibility studies. J Radiother Pract 2015;14(1):92-8. https://doi.org/10.1017/S1460396914000326.

[12] Fisher M, Applegate C, Ryalat M, et al. Evaluation of 3D printed immobilisation shells for head and neck IMRT. Open J Radiol 2014;4(4):322-8. https://doi.org/10. 4236/ojrad.2014.44042.

[13] Kim MJ, Lee SR, Lee MJ, Sohn JW, Yun HG, Choi JY, et al. Characterization of 3D printing techniques: toward patient specific quality assurance spine-shaped phantom for stereotactic body radiation therapy. PLoS ONE 2017;12(5):e0176227https://doi.org/10.1371/journal.pone.0176227.

[14] Ricotti R, Vavassori A, Bazani A, Ciardo D, Pansini F, Spoto R, et al. 3D-printed applicators for high dose rate brachytherapy: dosimetric assessment at different infill percentage. Phys Med 2016;32(12):1698-706. https://doi.org/10.1016/j. ejmp.2016.08.016.

[15] Ricotti R, Ciardo D, Pansini F, Bazani A, Comi S, Spoto R, et al. Dosimetric characterization of 3D printed bolus at different infill percentage for external photon beam radiotherapy. Phys Med 2017;39:25-32. https://doi.org/10.1016/j.ejmp. 2017.06.004.

[16] Dancewicz OL, Sylvander SR, Markwell TS, Crowe SB, Trapp JV. Radiological properties of 3D printed materials in kilovoltage and megavoltage photon beams. Phys Med 2017;38:111-8. https://doi.org/10.1016/j.ejmp.2017.05.051.

[17] GC Co., Exafine putty type. http://www.gcamerica.com/products/operatory/ EXAFAST/exafast_putty_ifu.pdf.

[18] Stratasys.com. Biocompatibile Clear MED610. http://www.stratasys.com/-/media/ files/material-spec-sheets/mds_pj_med610_0618a.pdf.

[19] Yu AW, Duncan JM, Dauka JS, Lewis A, Cobb J. A feasibility study into the use of three-dimensional printer modelling in acetabular fracture surgery. Adv Orthop 2015;2015:617046https://doi.org/10.1155/2015/617046.

[20] Mottini M, Jafari SS, Shafighi M, Schaller B. New approach for virtual surgical planning and mandibular reconstruction using a fibula free flap. Oral Oncol 2016;59:e6-9. https://doi.org/10.1016/j.oraloncology.2016.06.001.

[21] Reddy MV, Eachempati K, Gurava Reddy AV, Mugalur A. Novel application of 3D printing in brachytherapy using MED610 3D printed insert for I-125 ROPES eye plaque. Australas Phys Eng Sci Med 2016;39(4):863-70. https://doi.org/10.1007/ s13246-016-0480-8.

[22] Pixmeo Co., Osirix Lite. https://www.osirix-viewer.com.

[23] Visual Computing Lab. MeshLab. http://www.meshlab.net.

[24] Autodesk, Inc., Netfabb Basic 5.2. https://www.netfabb.com/file/546/download.

[25] Stratasys.com. Objet Studio. https://www.stratasys.com/3d-printers/objeteden260vs\#_ga $=2.195978602 .758442900 .1549091706-1944012151$. 1535266508 .

[26] Stratasys.com. PSS_PJ_ObjetEden260VS. http://www.stratasys.com/-/media/files/ printer-spec-sheets/pss_pj_objeteden260vs.pdf.

[27] Scott Crowe. Radiation Oncology Medical Physics Resources. CT density tables. http://sbcrowe.net/ct-density-tables/.

[28] Shimamoto H, Sumida I, Kakimoto N, Marutani K, Okahata R, Usami A, et al. Evaluation of the scatter doses in the direction of the buccal mucosa from dental metals. J Appl Clin Med Phys 2015;16(3):7374. https://doi.org/10.1120/jacmp. v16i3.5374. 